\title{
Serum Adipocytokines Levels and Their Association with Insulin Sensitivity in Morbidly Obese Individuals Undergoing Bariatric Surgery
}

\author{
Astha Sachan ${ }^{1}$, Archna Singh'1, Sakshi Shukla', Sandeep Aggarwal ${ }^{2}$, Ishfaq Mir', Rakhee Yadav',* \\ Departments of ${ }^{1}$ Biochemistry and ${ }^{2}$ Surgical Disciplines, All India Institute of Medical Sciences, New Delhi, India
}

Background: Obese adipose tissue secretes a variety of adipocytokines that act as metabolic regulators with complex mechanisms. Our objective was to compare serum concentration of a panel of adipocytokines between obese and non-obese individuals and identify any distinct patterns correlating with insulin sensitivity in obesity.

Methods: We designed a cross-sectional study among obese (body mass index [BMl] $\geq 30 \mathrm{~kg} / \mathrm{m}^{2}, \mathrm{n}=62$ ) and non-obese (BMl $<25 \mathrm{~kg} / \mathrm{m}^{2}, \mathrm{n}=32$ ) individuals to compare circulating levels of the adipokines, such as adiponectin and resistin in conjunction with the measurement of the levels of inflammatory cytokines including C-reactive protein (CRP), interleukin (IL)-6, IL-8, monocyte chemoattractant protein (MCP)-1, and tumor necrosis factor (TNF)- $\alpha$ using Luminex multiplex immunoassay with drop array technology. Correlations between circulating adipocytokine levels and those of multiple well-established markers of insulin resistance including homeostatic model assessment of insulin resistance (HOMA-IR), homeostatic model assessment of $\beta$-cell function (HOMA- $\beta$ ) and quantitative insulin sensitivity check index were also established.

Results: CRP, IL-8, MCP-1, and TNF- $\alpha$ levels were higher in obese than non-obese individuals; the CRP and IL-8 differences were statistically significant. CRP correlated significantly with markers of insulin resistance (fasting plasma insulin, HOMA-IR, and QUICKI), and adiponectin correlated with HOMA- $\beta$ in obese individuals. We divided the group of obese individuals on the basis of HOMA-IR levels into insulin-resistant (IR; HOMA-IR $\geq 2.5)$ and insulin-sensitive (IS; HOMA-IR <2.5) groups; and 43 out of 62 participants were IR despite comparable BMls. An overall proinflammatory profile was compared between IR and IS obese, though the values were higher in IR obese but the difference was not significant.

Conclusion: Obesity is associated with a general inflammatory milieu and a crosstalk between adipocytokines and insulin resistance is complex as well as multifactorial.

Key words: Obesity, Insulin resistance, Adipocytokines, Inflammation

\author{
Received August 21, 2020 \\ Reviewed September 29, 2020 \\ Accepted October 29, 2020 \\ ${ }^{*}$ Corresponding author \\ Rakhee Yadav \\ https://orcid.org/0000-0002-4405-9595 \\ Department of Biochemistry, \\ All India Institute of Medical Sciences, \\ New Delhi 110029, India \\ Tel: +91-8826640014 \\ E-mail: rakheeyadav@aiims.edu
}

\section{INTRODUCTION}

The rising incidence and prevalence of overweight and obesity are posing a major challenge to chronic disease prevention and public health across countries. ${ }^{1}$ Despite significant advances in our understanding of the underlying mechanisms of weight gain and associated comorbidities like insulin resistance and cardiovascular risk, the prevalence of obesity and metabolic syndrome is escalating at an unprecedented rate. ${ }^{2}$ Worldwide, obesity has nearly tripled since 1975. Presently, the majority of the world's population lives in countries in which obesity is responsible for more fatalities than malnutrition and underweight status. ${ }^{3}$ Overweight conditions and obesity, defined as a body mass index (BMI) of $\geq 30 \mathrm{~kg} / \mathrm{m}^{2}$, had highest prevalence (42.4\%) in the United States in 2017-2018. ${ }^{4}$ In-

Copyright @ 2020 Korean Society for the Study of Obesity

(a) This is an Open Access article distributed under the terms of the Creative Commons Attribution Non-Commercial License (https://creativecommons.org/licenses/by-nc/4.o/) which permits unrestricted non-commercial use, distribution, and reproduction in any medium, provided the original work is properly cited. 
dia is also witnessing a mounting prevalence of these conditions as more than 135 million individuals are affected by obesity, ${ }^{5}$ particularly abdominal obesity. Additionally, Asians often display higher fat percentages at similar BMIs when compared to their counterparts from the western world. ${ }^{6}$

The genesis of the adipose tissue dysfunction in obesity is complex. A review of the scientific literature suggests that in response to changes in the nutritional cues in the setting of varied genetic and environmental factors, the adipose tissue undergoes dynamic remodelling. ${ }^{2}$ This is evident in terms of quantitative and qualitative alterations in the population of adipocytes, thereby placing these cells under an immense metabolic and oxidative stress. ${ }^{2}$ This remodeling in adipose tissue is closely related to the changes in tissue functions like alterations in adipokine secretion, local hypoxia and adipocyte death, inflammation and worsening of metabolic health. ${ }^{7}$ Active secretion of a variety of adipocytokines, which modulate metabolic homeostasis, has been observed in obesity. ${ }^{8}$ These adipocytokines are measurable in the circulation and have been known to impart a systemic low-grade chronic inflammation profile to obese and overweight individuals. Adoption of a more comprehensive term, "meta-inflammation" or metabolically triggered inflammation, has been suggested which points towards the genesis of the inflammation. ${ }^{9}$ This inflammation is considered to be the major risk factor for the development of obesity-related comorbidities like insulin resistance and cardiovascular diseases. ${ }^{10}$

Many population-based studies performed to date have broadly stressed that an imbalance of pro- and anti-inflammatory adipocytokines impacts the metabolic outcomes in obesity in terms of associated comorbidities. ${ }^{7}$ However, the studies have not reported the levels of individual adipocytokines in obesity and metabolic syndrome consistently. This has made interpretation difficult, and the underlying reasons seem more complex than expected. ${ }^{11,12}$ An understanding of the inflammatory and metabolic crosstalk of the cytokines in the trajectory of obesity needs further exploration.

Therefore, we designed our study to compare the levels of adipocytokines in morbidly obese individuals undergoing bariatric surgery with those of non-obese individuals. We used a multiplexed array of the most potent adipokines known to affect insulin sensitivity, i.e., adiponectin and resistin, and other serum inflammatory cytokines like C-reactive protein (CRP), interleukins (IL)-6 and -8, monocyte chemoattractant protein (MCP)- 1 and tumor necrosis factor (TNF)- $\alpha$. We chose the Luminex multiplex immunoassay in combination with drop array technology for our study considering its advantages over conventional enzyme-linked immunosorbent assay (ELISA)..$^{13,14}$

Modulation of inflammatory status in obesity seems fundamental to its complex relationship with insulin response. Thus, by using the calculated parameters of insulin sensitivity including homeostatic model assessment of insulin resistance (HOMA-IR), ${ }^{15}$ the marker of homeostatic model assessment of $\beta$-cell function (HOMA- $\beta)^{16}$ and the quantitative insulin sensitivity check index (QUICKI), ${ }^{17}$ we aimed to establish an association between circulating adipocytokines and insulin sensitivity in obesity.

\section{METHODS}

\section{Study design and participant recruitment}

We designed an exploratory cross-sectional study in which participants were recruited through the elective surgery schedule of the Department of Surgical Disciplines (Laparoscopic and Bariatric Surgery), All India Institute of Medical Sciences (AIIMS), New Delhi. We included 62 adult individuals with obesity $\left(\mathrm{BMI} \geq 30 \mathrm{~kg} / \mathrm{m}^{2}\right)$ who were admitted for undergoing bariatric surgery. The control group included 32 non-obese (BMI $<25 \mathrm{~kg} / \mathrm{m}^{2}$ ) adult individuals undergoing other surgeries with associated disease conditions. These included laparoscopic cholecystectomy for cholelithiasis and incisional hernia repair. Individuals younger than 18 years and older than 60 years were excluded. We also excluded candidates diagnosed with diabetes mellitus and concomitant acute or chronic disorders of the immune system. Written informed consent was obtained from all participants, and the study was approved by the AIIMS Ethical Committee (A-536; IEC no. 473/01.09.2017, RP60/2017).

\section{Anthropometric measurements}

All subjects underwent comprehensive medical evaluation including history and physical examination prior to surgery. The age, sex, height $(\mathrm{cm})$, weight $(\mathrm{kg})$, and waist circumference $(\mathrm{cm})$ were recorded, and BMI was calculated as weight $(\mathrm{kg}) /$ height $\left(\mathrm{m}^{2}\right)$ for all participants. 


\section{Sample collection and processing}

Preoperative blood samples were obtained by venipuncture after overnight fasting in participants in both study groups. Appropriate vacutainer tubes were used for the measurement of plasma glucose, insulin and serum cytokines. After centrifugation at 3,000 rpm for 15 minutes, plasma and serum were separated. Plasma was used for measuring glucose and insulin, and serum was transferred and stored in sterile Eppendorf tubes at $-80^{\circ} \mathrm{C}$ for subsequent analysis.

\section{Plasma glucose and insulin assays and serum glycosylated hemoglobin measurement}

Plasma glucose was estimated using Randox GOD-PAP glucose estimation kit (Randox Laboratories, Crumlin, UK) according to manufacturer's protocol. ${ }^{18}$ Insulin was estimated using chemiluminescence-based immunoassay in a Liaison autoanalyzer (Diasorin, Saluggia, Italy). Serum glycosylated hemoglobin was determined using high performance liquid chromatography. ${ }^{18}$

To test for associations between the inflammation profile and insulin sensitivity status of the individuals in our study, we used the well-established markers of insulin resistance HOMA-IR, HOMA- $\beta$, and QUICKI as useful indices for insulin sensitivity. These indices are calculated parameters and use the value of fasting plasma glucose and insulin for their determination. HOMA-IR was calculated by using the formula $($ HOMA-IR $)=$ insulin $(\mu \mathrm{U} / \mathrm{mL}) \times[$ glucose $(\mathrm{mmol} / \mathrm{L}) / 22.5] \cdot{ }^{15}$ Low HOMA-IR $(<2.5)$ indicated insulin sensitivity, and high HOMA-IR values $(\geq 2.5$ ) indicated insulin resistance. ${ }^{18,19}$ We used this cutofflevel of HOMA-IR to further subgroup obese individuals as insulin-sensitive (IS) and insulin-resistant (IR) and compared the inflammatory status between the two. HOMA- $\beta$ was calculated as $20 \times$ insulin $(\mu \mathrm{U} / \mathrm{mL}) /[$ glucose $(\mathrm{mmol} / \mathrm{L})-3.5] .{ }^{16}$ QUICKI was calculated as $1 /\left[\log \left(\mathrm{I}_{0}\right)+\log \left(\mathrm{G}_{0}\right)\right]$, where $\mathrm{I}_{0}$ is fasting insulin $(\mu \mathrm{U} / \mathrm{mL})$ and $\mathrm{G}_{0}$ is fasting glucose $(\mathrm{mg} / \mathrm{dL}) .{ }^{17}$ QUICKI is one of the most accurate and useful indices for determining insulin sensitivity; a value of below 0.34 suggests insulin resistance and below 0.30 supports the diagnosis of diabetes mellitus. ${ }^{17}$

\section{Serum adipocytokine assay}

Serum samples were subjected to quantitative analysis of the circulating adipocytokines. A panel was made for measuring serum adipokines and inflammatory cytokines. This panel consisted of adiponectin, resistin, CRP, IL-8, MCP-1, IL-6, and TNF- $\alpha$. These were assayed using R\&D Systems Luminex Multiplex Immunoassay (Minneapolis, MN, USA) as per the manufacturer's protocol in combination with Drop array technology and read in a Bio-Plex 200 reader (Bio-Rad, Hercules, CA, USA). This system makes use of xMAP magnetic bead microspheres which act as both the identifier and the solid surface to build the assay. In this system, multiplex immunoassays consisting of analyte-specific capture antibodies are conjugated to XMAP beads. A combination of analyzer and software for data acquisition and analysis allows higher throughput and accuracy than conventional immunoassays with sample volumes as low as $25 \mu \mathrm{L}$. Multiplexing also minimizes reaction to reaction inconsistencies as might be seen with a separate ELISA for each cytokine. ${ }^{13,14}$

\section{Statistical methods}

To describe patient demographics and anthropometric characteristics and biochemical parameters, the data was summarized and analyzed using Prism version 8.0.1 (GraphPad, San Diego, CA, USA). Data were tested for normality using Shapiro-Wilk/KolmogorovSmirnov test. Continuous data was expressed as mean \pm standard error of the mean. Student $t$-test was used to compare the normally-distributed variables between study groups, and Mann-Whitney U-test was performed to compare the non-normally-distributed variables. A $P$-value of less than 0.05 was considered to be statistically significant. Correlation between anthropometric characteristics and markers of insulin resistance with the levels of circulating adipocytokines was calculated using Spearman's correlation coefficient. The $P$-values $<0.05$ were considered to be statistically significant.

\section{RESULTS}

\section{Clinical characteristics of participants}

Average BMI of the individuals in the obese study group was $45.76 \pm 0.81 \mathrm{~kg} / \mathrm{m}^{2}$ and in the non-obese control group $24.19 \pm$ $0.80 \mathrm{~kg} / \mathrm{m}^{2}$. Average age of individuals in the obese and non-obese group was $39.55 \pm 1.38$ and $38.91 \pm 2.63$ years, respectively (Table 1 ). The sex distribution was 53 females and 9 males in the obese group and 20 females and 12 males in the non-obese group. 


\section{Biochemical parameters and markers of insulin resistance}

All individuals in our study were non-diabetic, but we found significantly higher fasting plasma glucose and insulin levels in obese as compared to non-obese participants. All markers of insulin resistance, i.e., HOMA-IR, HOMA- $\beta$, and QUICKI, showed that the participants in the obese group were significantly more insulin-resistant than those in the non-obese group (Table 1). Since one of the objectives of our study was to assess how inflammatory status differed with the insulin response in obese individuals, we divided the participants in this group into IS and IR obese subgroups based on the HOMA-IR cutoff as previously mentioned in the Methods section. ${ }^{18,19}$ Out of 62 obese individuals in our study, 43 were IR and 19 were IS (Table 2). The difference in their BMI was not significant; however, all three insulin sensitivity markers, i.e., HOMAIR, HOMA- $\beta$, and QUICKI, showed significant differences between IR and IS obese groups (Table 2).

Table 1. Anthropometric characteristics, biochemical parameters and serum adipocytokines in obese and non-obese groups

\begin{tabular}{|c|c|c|c|}
\hline Variable & Obese $(n=62)$ & Non-obese $(n=32)$ & $P$ \\
\hline Age $(y r)^{*}$ & $39.55 \pm 1.38$ & $38.91 \pm 2.63$ & NS \\
\hline \multicolumn{4}{|l|}{ Anthropometric characteristics* } \\
\hline Body weight (kg) & $115.8 \pm 3.06$ & $61.14 \pm 2.01$ & $<0.001$ \\
\hline $\mathrm{BMI}\left(\mathrm{kg} / \mathrm{m}^{2}\right)$ & $45.76 \pm 0.81$ & $24.19 \pm 0.70$ & $<0.001$ \\
\hline \multicolumn{4}{|l|}{ Clinical parameter* } \\
\hline Fasting plasma glucose (mg/dL) & $107.1 \pm 3.02$ & $95.26 \pm 2.81$ & 0.006 \\
\hline Fasting plasma insulin $(\mu \mathrm{U} / \mathrm{L})$ & $21.86 \pm 2.36$ & $10.73 \pm 1.67$ & $<0.001$ \\
\hline HOMA-IR & $5.38 \pm 0.65$ & $2.17 \pm 0.24$ & $<0.001$ \\
\hline НОМА- $\beta$ & $242.4 \pm 33.45$ & $115.3 \pm 18.56$ & 0.001 \\
\hline QUICKI & $0.31 \pm 0.00$ & $0.48 \pm 0.12$ & 0.035 \\
\hline \multicolumn{4}{|l|}{ Serum adipocytokine $^{\dagger}$} \\
\hline $\mathrm{CRP}(\mu \mathrm{g} / \mathrm{mL})$ & $52.56 \pm 15.55$ & $34.05 \pm 19.09$ & 0.006 \\
\hline Adiponectin $(\mu \mathrm{g} / \mathrm{mL})$ & $39.88 \pm 4.17$ & $44.09 \pm 7.33$ & 0.593 \\
\hline IL-8 (pg/mL) & $25.80 \pm 38.45$ & $36.39 \pm 6.45$ & 0.025 \\
\hline MCP-1 (ng/mL) & $1.090 \pm 0.17$ & $0.75 \pm 0.05$ & 0.063 \\
\hline Resistin (ng/mL) & $39.70 \pm 5.98$ & $36.53 \pm 5.86$ & 0.707 \\
\hline IL-6 (pg/mL) & $26.88 \pm 6.99$ & $37.72 \pm 32.72$ & $<0.001$ \\
\hline TNF- $\alpha(p g / m L)$ & $38.20 \pm 7.88$ & $23.05 \pm 3.63$ & 0.084 \\
\hline
\end{tabular}

Values are presented as mean \pm standard error of the mean.

The $P$-values obtained by using * Student t-test (unpaired) for parametric; ${ }^{\dagger}$ Mann-Whitney U-test for non-parametric variables between two groups $(P<0.05$ is considered significant).

NS, not significant; BMI, body mass index; HOMA-IR, homeostatic model assessment of insulin resistance; HOMA- $\beta$, homeostatic model assessment of $\beta$-cell function; QUICKI, quantitative insulin sensitivity check index; CRP, C-reactive protein; IL, interleukin; MCP, monocyte chemoattractant protein; TNF, tumor necrosis factor.

\section{Serum adipocytokine assay}

Out of the five inflammatory cytokines that we assayed in our study, we found a higher level of CRP, IL-8, MCP-1, and TNF- $\alpha$ in obese as compared to non-obese individuals; the difference, however, was significant only for CRP and IL-8 (Table 1). Surprisingly, IL-6 was found to be significantly lower in obese than in non-obese individuals, contrary to the data generally reported in most of the literature. ${ }^{2,20}$ Considering the two adipokines that were part of the same array, resistin was higher and adiponectin was lower in obese participants than in non-obese ones; the differences, however, were not statistically significant (Table 1).

Our next aim was to assess how the inflammatory status with obesity changed with whole body insulin resistance. We found that neither of the adipocytokines showed a significant difference between IR and IS obese groups. Overall, though, a presence of increased proinflammatory profile was evident for the IR obese par-

Table 2. Anthropometric characteristics, biochemical parameters and serum adipocytokines in IR obese and IS obese subgroups

\begin{tabular}{|c|c|c|c|}
\hline Variable & IR obese (n=43) & IS obese $(n=19)$ & $P$ \\
\hline $\operatorname{Age}(y r)^{*}$ & $39.07 \pm 1.66$ & $40.63 \pm 2.53$ & NS \\
\hline \multicolumn{4}{|l|}{ Anthropometric characteristics* } \\
\hline Body weight (kg) & $116.40 \pm 3.92$ & $114.30 \pm 4.44$ & 0.762 \\
\hline $\mathrm{BMI}\left(\mathrm{kg} / \mathrm{m}^{2}\right)$ & $46.20 \pm 1.01$ & $44.66 \pm 1.34$ & 0.399 \\
\hline \multicolumn{4}{|l|}{ Clinical parameter* } \\
\hline Fasting serum glucose (mg/dL) & $107.40 \pm 3.76$ & $106.20 \pm 5.02$ & 0.862 \\
\hline Fasting serum insulin ( $\mu \mathrm{U} / \mathrm{L})$ & $25.74 \pm 2.83$ & $9.949 \pm 1.76$ & $0.003^{\dagger}$ \\
\hline HOMA-IR & $6.53 \pm 0.74$ & $1.35 \pm 0.15$ & $<0.001$ \\
\hline HOMA- $\beta$ & $284.40 \pm 40.37$ & $95.40 \pm 21.72$ & 0.017 \\
\hline QUICKI & $0.30 \pm 0.003$ & $0.35 \pm 0.01$ & $<0.001$ \\
\hline $\mathrm{HbA1c}$ & $6.07 \pm 0.09$ & $5.83 \pm 0.26$ & 0.297 \\
\hline \multicolumn{4}{|l|}{ Serum adipocytokines ${ }^{\dagger}$} \\
\hline $\mathrm{CRP}(\mu \mathrm{g} / \mathrm{mL})$ & $60.79 \pm 20.04$ & $33.90 \pm 22.92$ & 0.430 \\
\hline Adiponectin ( $\mu \mathrm{g} / \mathrm{mL})$ & $52.16 \pm 6.61$ & $72.17 \pm 12.27$ & 0.124 \\
\hline IL-8 (pg/mL) & $152.40 \pm 53.40$ & $65.82 \pm 31.69$ & 0.179 \\
\hline MCP-1 (ng/mL) & $1.05 \pm 0.16$ & $1.18 \pm 0.43$ & 0.177 \\
\hline Resistin (ng/mL) & $38.37 \pm 6.60$ & $42.71 \pm 12.8$ & 0.741 \\
\hline IL-6 (pg/mL) & $32.21 \pm 9.70$ & $14.82 \pm 5.64$ & 0.091 \\
\hline TNF- $\alpha(p g / m L)$ & $35.45 \pm 6.49$ & $44.42 \pm 21.46$ & 0.510 \\
\hline
\end{tabular}

Values are presented as mean \pm standard error of the mean.

The $P$-values obtained by using *Student t-test (unpaired) for parametric; ${ }^{\dagger}$ Mann-Whitney U-test for non-parametric variables between two groups $(P<0.05$ is considered significant).

IR, insulin-resistant; IS, insulin-sensitive; NS, not significant; BMI, body mass index; HOMA-IR, homeostatic model assessment of insulin resistance; HOMA- $\beta$, homeostatic model assessment of $\beta$-cell function; QUICKI, quantitative insulin sensitivity check index; HbA1c, glycosylated hemoglobin; CRP, C-reactive protein; IL, interleukin; MCP monocyte chemoattractant protein; TNF, tumor necrosis factor. 
ticipants due to their higher levels of CRP, IL-8, and IL-6 than the IS obese group participants. This finding suggests that despite being non-diabetic clinically, 43 out of 62 of the IR obese individuals presented with a proinflammatory profile (Table 2 ). Those in this group are at higher risk of developing metabolic and cardiovascular complications.

\section{Correlation of inflammatory profile of obese with} anthropometric characteristics and markers of insulin resistance

The analysis of correlations was done using Spearman's correlation coefficient because many variables were not normally-distributed in different groups. Out of the cytokines studied, IL-8 correlated significantly with BMI of obese $(r=0.215, P=0.04)$ and IS

Table 3. Correlations among circulating adipocytokines, anthropometric characteristics and markers of insulin resistance in obese individuals undergoing bariatric surgery

\begin{tabular}{lccccrrrrr}
\hline Variable & Weight & BMI & $\begin{array}{c}\text { Fasting plasma } \\
\text { glucose }\end{array}$ & $\begin{array}{c}\text { Fasting plasma } \\
\text { insulin }\end{array}$ & QUICKI & HOMA-IR & HOMA- $\beta$ & HbA1C \\
\hline CRP & -0.099 & 0.030 & -0.035 & $0.289^{*}$ & $-0.239^{*}$ & $0.382^{\dagger}$ & 0.150 & 0.038 \\
Adiponectin & -0.145 & -0.051 & $0.305^{*}$ & 0.034 & -0.204 & $0.251^{*}$ & -0.134 & -0.042 \\
IL-8 & 0.132 & $0.215^{*}$ & 0.078 & 0.008 & -0.018 & 0.203 & -0.001 & 0.122 \\
MCP-1 & -0.010 & 0.104 & -0.099 & 0.043 & 0.005 & -0.018 & 0.053 & -0.049 \\
Resistin & 0.004 & -0.175 & -0.137 & -0.012 & 0.115 & -0.106 & 0.082 & -0.177 \\
IL-6 & 0.016 & 0.069 & -0.205 & 0.104 & -0.061 & 0.081 & 0.153 & 0.186 \\
TNF- $\alpha$ & -0.107 & 0.015 & $0.262^{*}$ & 0.118 & -0.136 & 0.185 & -0.082 & 0.054 \\
\hline
\end{tabular}

Spearman correlation coefficient represented as r-value: ${ }^{*} P<0.05{ }^{\dagger} P<0.01$.

$\mathrm{BMI}$, body mass index; QUICKI, quantitative insulin sensitivity check index; HOMA-IR, homeostatic model assessment of insulin resistance; HOMA- $\beta$, homeostatic model assessment of $\beta$-cell function; HbA1c, glycosylated hemoglobin; CRP, C-reactive protein; IL, interleukin; MCP, monocyte chemoattractant protein; TNF, tumor necrosis factor.

Table 4. Correlations among circulating adipocytokines with anthropometric characteristics and markers of insulin resistance in IR and IS obese individuals undergoing bariatric surgery

\begin{tabular}{|c|c|c|c|c|c|c|c|c|}
\hline Variable & Weight & $\mathrm{BMI}$ & $\begin{array}{l}\text { Fasting plasma } \\
\text { glucose }\end{array}$ & $\begin{array}{l}\text { Fasting plasma } \\
\text { insulin }\end{array}$ & QUICKI & HOMA-IR & HOMA- $\beta$ & $\mathrm{HbA1c}$ \\
\hline \multicolumn{9}{|l|}{ CRP } \\
\hline $\mathbb{R}$ & -0.010 & 0.013 & 0.081 & $0.282^{*}$ & $-0.306^{*}$ & $0.380^{\dagger}$ & 0.104 & 0.016 \\
\hline IS & -0.308 & 0.046 & -0.365 & 0.039 & 0.281 & $0.678^{\dagger}$ & -0.034 & 0.025 \\
\hline \multicolumn{9}{|c|}{ Adiponectin } \\
\hline $\mathbb{R}$ & -0.158 & - & $0.503^{\dagger}$ & -0.063 & -0.171 & 0.160 & $-0.323^{*}$ & 0.004 \\
\hline IS & -0.134 & -0.301 & -0.344 & 0.164 & -0.277 & $0.545^{*}$ & 0.433 & 0.088 \\
\hline \multicolumn{9}{|l|}{ IL-8 } \\
\hline $\mathbb{R}$ & 0.018 & 0.040 & 0.113 & -0.145 & 0.084 & - & -0.101 & 0.206 \\
\hline IS & 0.394 & $0.483^{*}$ & -0.049 & -0.048 & 0.288 & 0.165 & 0.010 & -0.177 \\
\hline \multicolumn{9}{|l|}{ MCP-1 } \\
\hline $\mathbb{R}$ & -0.107 & 0.003 & -0.161 & -0.069 & 0.120 & -0.112 & 0.053 & -0.085 \\
\hline IS & 0.188 & 0.369 & 0.157 & -0.090 & 0.327 & -0.006 & -0.174 & -0.060 \\
\hline \multicolumn{9}{|l|}{ Resistin } \\
\hline $\mathbb{R}$ & -0.204 & $-0.419^{\dagger}$ & -0.176 & 0.032 & 0.103 & -0.024 & 0.146 & -0.146 \\
\hline IS & $0.532^{*}$ & $0.564^{*}$ & 0.085 & 0.129 & 0.145 & -0.062 & 0.111 & -0.155 \\
\hline \multicolumn{9}{|l|}{ IL-6 } \\
\hline $\mathbb{R}$ & 0.031 & - & -0.156 & -0.012 & -0.007 & -0.025 & 0.125 & 0.204 \\
\hline IS & -0.075 & 0.087 & $-0.436^{*}$ & -0.020 & 0.410 & $-0.504^{*}$ & 0.192 & 0.018 \\
\hline \multicolumn{9}{|l|}{ TNF- $\alpha$} \\
\hline $\mathbb{R}$ & -0.157 & -0.009 & $0.312^{*}$ & -0.130 & 0.053 & 0.058 & -0.206 & 0.093 \\
\hline IS & 0.025 & -0.079 & 0.091 & 0.453 & -0.233 & 0.229 & 0.129 & -0.074 \\
\hline
\end{tabular}

Spearman correlation coefficient represented as r-value: ${ }^{*} P<0.05 ;{ }^{\dagger} P<0.01$

IR, insulin-resistant; IS, insulin-sensitive; BMI, body mass index; QUICKI, quantitative insulin sensitivity check index; HOMA-IR, homeostatic model assessment of insulin resistance; HOMA- $\beta$, homeostatic model assessment of $\beta$-cell function; HbA1c, glycosylated hemoglobin; CRP, C-reactive protein; IL, interleukin; MCP, monocyte chemoattractant protein; TNF, tumor necrosis factor. 
obese individuals $(\mathrm{r}=0.483, P=0.02)$. Only CRP correlated significantly with fasting insulin, HOMA-IR and QUICKI in obese and IR obese individuals. Also, CRP was the only cytokine that correlated significantly with HOMA-IR in obese $(\mathrm{r}=0.382, P=0.002)$, IR obese $(\mathrm{r}=0.380, P=0.006)$ and IS obese $(\mathrm{r}=0.678, P=0.009)$ groups and subgroups respectively. There was also a significant positive correlation between TNF- $\alpha$ and fasting plasma glucose in the obese group $(\mathrm{r}=0.262, P=0.02)$ and IR obese groups $(\mathrm{r}=0.312$, $P=0.02$ ) (Tables 3 and 4 ). Therefore the overall proinflammatory milieu in obesity correlated significantly with insulin resistance.

With respect to the two adipokines studied in our participants, adiponectin correlated significantly with pancreatic insulin secretion capacity demonstrated by its negative correlation with HOMA- $\beta$ in the IR obese subgroup $(\mathrm{r}=-0.323, P=0.01)$ (Table 4). Resistin showed a significant positive correlation with weight $(\mathrm{r}=0.532$, $P=0.014)$ and BMI $(\mathrm{r}=0.564, P=0.01)$ in the IS obese group but an unexpected negative correlation with BMI $(\mathrm{r}=-0.419, P=0.002)$ in the IR obese (Tables 3 and 4).

\section{DISCUSSION}

In our study, we sought to evaluate the distinct pattern of an array of circulating adipocytokines in obese as compared to nonobese individuals. We designed a cross-sectional study in which we also intended to establish a correlation between the inflammatory profile of the obese subjects and insulin response. All participants in our study were non-diabetic, but the markers of insulin resistance (HOMA-IR, HOMA- $\beta$, and QUICKI) significantly showed that obese individuals were more insulin-resistant than non-obese ones (Table 2). We measured two important adipokines (adiponectin and resistin) in all participants of our study and considered their opposite roles in mediating the insulin response; adiponectin has been implicated in increasing insulin sensitivity, and resistin has been linked to obesity with insulin resistance. ${ }^{21,22}$ We found that obese individuals had lower adiponectin levels than non-obese ones. The preponderance of scientific evidence suggests that adiponectin level has an inverse association with adiposity, and higher levels of adiponectin have been observed in lean individuals. ${ }^{23}$ In addition, many studies have shown that adiponectin level is lower in the insulin-resistant state and increases with improvement in the insulin sensitivity associated with the weight loss. ${ }^{21}$ We also found a lower level of adiponectin in IR obese individuals as compared to IS obese ones in our study, but the difference was not statistically significant (Table 2). However, adiponectin correlated significantly with HOMA- $\beta$ in IR obese individuals, but its correlation with other markers of insulin resistance was inconclusive across the groups in our study (Tables 3 and 4). Similar observations were made in other studies, and an "adiponectin paradox" in which epidemiological data demonstrated that increased adiponectin levels correlated with higher cardiovascular or all-cause mortality was reported. ${ }^{24}$ The underlying mechanism behind this paradox is still unclear, but one hypothesis is that there may be a compensatory elevation of adiponectin in patients with metabolic abnormalities. This compensatory elevation may be associated with higher future mortality. ${ }^{24}$

The other adipokine analyzed in our study was resistin which has an opposite effect as that of adiponectin in terms of insulin sensitivity. Experimental studies have shown that resistin increases hepatic gluconeogenesis and insulin resistance. ${ }^{25}$ We did not find a significant difference in the levels of this adipokine in either obese vs. non-obese or IR versus IS obese groups. Population-based studies have shown that the association between resistin levels and insulin sensitivity has been inconsistent. ${ }^{24}$ Furthermore, resistin's role in insulin resistance and glucose metabolism has been inconclusive in human studies. ${ }^{26}$ The only significant correlations that we found for resistin were with weight and BMI, and those associations were only in the IS obese group. This finding is in agreement with several studies reporting that increased plasma resistin levels were associated with increased BMI. ${ }^{26}$

Apart from their opposite roles in mitigating insulin response, both adiponectin and resistin also have opposite effects on inflammation profiles. Adiponectin has anti-inflammatory and anti-atherogenic effects ${ }^{21}$ by suppressing proinflammatory cytokines such as TNF- $\alpha$ and by inducing other anti-inflammatory factors. ${ }^{21} \mathrm{How}-$ ever, resistin has been shown to exert proinflammatory effects by increasing the production of IL- 6 and TNF- $\alpha .{ }^{27}$

We included inflammatory cytokines in the array of adipocytokines in our study and found that CRP, IL-8, MCP-1, and TNF- $\alpha$ were higher in obese than non-obese participants. CRP and IL-8 level were significantly different suggesting that obesity is a chronic 
inflammatory state. However, IL-6, despite being proinflammatory, was found to be significantly lower in obese than non-obese individuals in our study. There are several potential explanations for this ostensibly contradictory data for the role of IL-6 in glucose metabolism and insulin action. ${ }^{10}$ Researchers disagreed on whether IL-6 correlated with BMI, insulin levels or HOMA-IR. ${ }^{28}$ Many experimental studies have determined that there exists a fine-tuned balance between anti-and proinflammatory actions of IL- $6 .{ }^{29}$ Additionally, IL-6 is produced by different organs. This may contribute to the layers of complexity associated with its actions on metabolic regulation. ${ }^{10}$ Owing to such inconsistencies, a pleiotropic functional profile has been suggested for IL- $6{ }^{30}$

Another IL measured in our study was IL-8, and we found significantly higher levels of IL-8 in obese than in non-obese individuals. This also applied to the IR versus IS obese subgroups. IL-8 also showed a significant positive correlation with BMI in both obese and IR obese groups. Some of the recent studies have clearly demonstrated that higher IL-8 levels are associated with obesity. ${ }^{13} \mathrm{IL}-8$ levels are also closely correlated with obesity-related parameters like BMI, waist circumference, CRP, IL-6 levels and HDL cholesterol levels. ${ }^{31}$

One of the important and most abundant immune cells infiltrating the adipose tissue in obesity are macrophages, ${ }^{10}$ and MCP-1 is an important chemoattractant chemokine produced by adipose tissue mediating macrophage recruitment. ${ }^{2}$ We found increased circulating levels of MCP-1 in obese individuals as compared to nonobese ones, but the difference was not statistically significant. Dahlman et al..$^{32}$ found that expression of MCP-1 is significantly higher in obese as compared to lean individuals, but circulating levels showed no changes among their study groups suggesting that obesity produced only local changes of MCP-1 in adipose tissue.

There is putative evidence that an imbalance of the pro-and antiinflammatory adipokines secreted by adipose tissue is one of the major contributors to metabolic dysfunction. However, there are inconsistencies in the outcomes of a number of human studies. ${ }^{11,33}$ We did not find a significant difference but observed a notable trend in which classical proinflammatory cytokines like CRP, IL-8, and IL- 6 were found to be higher and MCP- 1 and TNF- $\alpha$ were paradoxically lower in IR as compared to IS obese study participants.

TNF- $\alpha$ has been the most consistently studied inflammatory cy- tokine in obesity ever since its identification in the adipose tissue of obese mice. This marked the start of the metabolic inflammation concept. ${ }^{10,34}$ TNF- $\alpha$ levels did not show any significant difference between the groups, but correlated significantly with fasting plasma glucose in obese and IR obese individuals in our study. Strong associations have been shown in several human studies between circulating TNF- $\alpha$ and insulin resistance ${ }^{35}$ or other metabolic complications. ${ }^{36}$ However, attempts to block TNF- $\alpha$ functions have never produced consistent metabolic outcomes. ${ }^{10}$ Adipose tissue does not significantly contribute to circulating TNF- $\alpha$ levels. ${ }^{37}$ In fact, the soluble forms of the receptor of TNF- $\alpha$ (TNFR2) is secreted from adipose tissue; this may modulate the actions of TNF- $\alpha$ and warrants detailed exploration. ${ }^{37}$

Functional pleiotropy and redundancy are characteristic features of cytokines. As cytokines are produced by many different cell types, cytokines often show overlapping activities in regulating proliferation or differentiation; these regulatory activities depend on the type and developmental state of the target cells involved. ${ }^{30}$ Collectively, the signaling of TNF- $\alpha$ and IL- 6 in obesity-associated inflammation is complex, especially in developing concomitant insulin resistance, owing to their temporal and cell-type specific functions. ${ }^{29}$ Considering that resistin increases IL- 6 and TNF- $\alpha$ secretion along with that of other adhesion molecules, ${ }^{24}$ we may assume that inconsistencies in resistin levels may also be responsible for unexpected results and correlations for these two cytokines.

Fundamental differences in mechanisms that regulate inflammatory cytokines (TNF- $\alpha, \mathrm{IL}-6$, and IL- 8 ) and acute phase proteins like CRP may explain the inconsistency of relationships between these markers and metabolic phenotypes in morbidly obese individuals. ${ }^{20,28}$ Equally possible is that technical differences in sample collection/handling may be responsible for varying outcomes.

Of all the cytokines we studied, only CRP correlated significantly with most of the markers of insulin resistance (HOMA-IR, QUICKI, and fasting insulin) and had significantly higher levels in obese and IR obese individuals. CRP is the most often measured inflammatory marker and has consistently correlated with weight, BMI and waist to hip ratio as well as insulin resistance, ${ }^{2,20} \mathrm{CRP}$ has become the marker of metainflammation. ${ }^{2}$ This may be attributed to its crosstalk with other cytokines like TNF- $\alpha$ and IL-6.,20 Despite the extensive characterization of the mechanisms by which inflamma- 
tory cytokines promote insulin resistance and adipose tissue dysfunctions, targeted immunotherapies have not yielded any positive outcome. ${ }^{11}$ Driving forces behind changes in inflammatory markers are multifactorial. A chronology of the triggers for each of the cytokines that can better establish the trajectory of presentation and inter- and intra-individual variations is lacking.

Based on the available literature and the findings of our study, obesity is associated with a general inflammatory response that cannot be simply attributed to mere imbalance between pro- and anti-inflammatory status. A comprehensive understanding of the crosstalk between inflammation and insulin response with the quantum of effect of individual cytokines is necessary to provide a better insight into the functions of adipose tissue as an essential metabolic regulator.

\section{CONFLICTS OF INTEREST}

The authors declare no conflict of interest.

\section{ACKNOWLEDGMENTS}

This work was funded by Intramural Research Grant (Project code. A-536), by All India Institute of Medical Sciences. We thank SA for providing, and Mr. Mudassir for collecting patient samples from the Department of Surgical Disciplines. We also thank Dr. Sivasankar Baalasubramanian (Executive Director, Indoor Biotechnologies India Pvt. Ltd., Bangalore) for analyzing the samples for the planned array of cytokines.

\section{AUTHOR CONTRIBUTIONS}

Study concept and design: RY, SA, and AS (Archna Singh); acquisition of data: AS (Astha Sachan), SS, and IM; analysis and interpretation of data: RY and AS (Archna Singh); drafting of the manuscript: RY and AS (Astha Sachan); critical revision of the manuscript: RY, SA, and AS (Archna Singh); statistical analysis: AS (Astha Sachan); obtained funding: RY; administrative, technical, or material support: RY; and study supervision: RY.

\section{REFERENCES}

1. Hruby A, Hu FB. The epidemiology of obesity: a big picture. Pharmacoeconomics 2015;33:673-89.

2. Phillips CL, Grayson BE. The immune remodel: weight lossmediated inflammatory changes to obesity. Exp Biol Med (Maywood) 2020;245:109-21.

3. World Health Organization. Obesity and overweight [Internet]. Geneva: World Health Organization; 2020 [cited 2020 Oct 29]. Available from: https://www.who.int/news-room/ fact-sheets/detail/obesity-and-overweight

4. Centers for Disease Control and Prevention. Overweight and obesity [Internet]. Atlanta (GA): Centers for Disease Control and Prevention; 2020 [cited 2020 Oct 29]. Available from: https://www.cdc.gov/obesity/data/adult.html

5. Ahirwar R, Mondal PR. Prevalence of obesity in India: a systematic review. Diabetes Metab Syndr 2019;13:318-21.

6. Sattar N, McInnes IB, McMurray JJ. Obesity is a risk factor for severe COVID-19 infection: multiple potential mechanisms. Circulation 2020;142:4-6.

7. Choe SS, Huh JY, Hwang IJ, Kim JI, Kim JB. Adipose tissue remodeling: its role in energy metabolism and metabolic disorders. Front Endocrinol (Lausanne) 2016;7:30.

8. Wang Z, Nakayama T. Inflammation, a link between obesity and cardiovascular disease. Mediators Inflamm 2010;2010: 535918.

9. Hotamisligil GS. Inflammation and metabolic disorders. Nature 2006;444:860-7.

10. Cao H. Adipocytokines in obesity and metabolic disease. J Endocrinol 2014;220:T47-59.

11. Rakotoarivelo V, Lacraz G, Mayhue M, Brown C, Rottembourg D, Fradette J, et al. Inflammatory cytokine profiles in visceral and subcutaneous adipose tissues of obese patients undergoing bariatric surgery reveal lack of correlation with obesity or diabetes. EBioMedicine 2018;30:237-47.

12. Zhang C, Zhang J, Liu Z, Zhou Z. More than an anti-diabetic bariatric surgery, metabolic surgery alleviates systemic and local inflammation in obesity. Obes Surg 2018;28:3658-68.

13. Harakeh S, Kalamegam G, Pushparaj PN, Al-Hejin A, Alfadul SM, Al Amri T, et al. Chemokines and their association with 
body mass index among healthy Saudis. Saudi J Biol Sci 2020; 27:6-11.

14. Dalmas E, Rouault C, Abdennour M, Rovere C, Rizkalla S, Bar-Hen A, et al. Variations in circulating inflammatory factors are related to changes in calorie and carbohydrate intakes early in the course of surgery-induced weight reduction. Am J Clin Nutr 2011;94:450-8.

15. Matthews DR, Hosker JP, Rudenski AS, Naylor BA, Treacher DF, Turner RC. Homeostasis model assessment: insulin resistance and beta-cell function from fasting plasma glucose and insulin concentrations in man. Diabetologia 1985;28:412-9.

16. Sung KC, Reaven GM, Kim SH. Utility of homeostasis model assessment of beta-cell function in predicting diabetes in 12,924 healthy Koreans. Diabetes Care 2010;33:200-2.

17. Katz A, Nambi SS, Mather K, Baron AD, Follmann DA, Sullivan G, et al. Quantitative insulin sensitivity check index: a simple, accurate method for assessing insulin sensitivity in humans. J Clin Endocrinol Metab 2000;85:2402-10.

18. Vincent V, Thakkar H, Aggarwal S, Mridha AR, Ramakrishnan L, Singh A. ATP-binding cassette transporter A1 (ABCA1) expression in adipose tissue and its modulation with insulin resistance in obesity. Diabetes Metab Syndr Obes 2019;12: 275-84.

19. Singh Y, Garg MK, Tandon N, Marwaha RK. A study of insulin resistance by HOMA-IR and its cut-off value to identify metabolic syndrome in urban Indian adolescents. J Clin Res Pediatr Endocrinol 2013;5:245-51.

20. Askarpour M, Khani D, Sheikhi A, Ghaedi E, Alizadeh S. Effect of bariatric surgery on serum inflammatory factors of obese patients: a systematic review and meta-analysis. Obes Surg 2019;29:2631-47.

21. Hosseinzadeh-Attar MJ, Golpaie A, Janani L, Derakhshanian $H$. Effect of weight reduction following bariatric surgery on serum visfatin and adiponectin levels in morbidly obese subjects. Obes Facts 2013;6:193-202.

22. Steppan CM, Bailey ST, Bhat S, Brown EJ, Banerjee RR, Wright CM, et al. The hormone resistin links obesity to diabetes. Nature 2001;409:307-12.

23. Fantuzzi G. Adiponectin and inflammation: consensus and controversy. J Allergy Clin Immunol 2008;121:326-30.
24. Choi SH, Hong ES, Lim S. Clinical implications of adipocytokines and newly emerging metabolic factors with relation to insulin resistance and cardiovascular health. Front Endocrinol (Lausanne) 2013;4:97.

25. Steppan CM, Lazar MA. Resistin and obesity-associated insulin resistance. Trends Endocrinol Metab 2002;13:18-23.

26. Goktas Z, Moustaid-Moussa N, Shen CL, Boylan M, Mo H, Wang S. Effects of bariatric surgery on adipokine-induced inflammation and insulin resistance. Front Endocrinol (Lausanne) 2013;4:69.

27. Singla P, Bardoloi A, Parkash AA. Metabolic effects of obesity: a review. World J Diabetes 2010;1:76-88.

28. Hafida S, Mirshahi T, Nikolajczyk BS. The impact of bariatric surgery on inflammation: quenching the fire of obesity? Curr Opin Endocrinol Diabetes Obes 2016;23:373-8.

29. Kern L, Mittenbühler MJ, Vesting AJ, Ostermann AL, Wunderlich CM, Wunderlich FT. Obesity-induced TNF $\alpha$ and IL-6 signaling: the missing link between obesity and inflammation-driven liver and colorectal cancers. Cancers (Basel) 2018;11:24.

30. Scheller J, Chalaris A, Schmidt-Arras D, Rose-John S. The pro- and anti-inflammatory properties of the cytokine interleukin-6. Biochim Biophys Acta 2011;1813:878-88.

31. Kim CS, Park HS, Kawada T, Kim JH, Lim D, Hubbard NE, et al. Circulating levels of MCP-1 and IL-8 are elevated in human obese subjects and associated with obesity-related parameters. Int J Obes (Lond) 2006;30:1347-55.

32. Dahlman I, Kaaman M, Olsson T, Tan GD, Bickerton AS, Wåhlén $\mathrm{K}$, et al. A unique role of monocyte chemoattractant protein 1 among chemokines in adipose tissue of obese subjects. J Clin Endocrinol Metab 2005;90:5834-40.

33. Lim S, Shin SM, Nam GE, Jung CH, Koo BK. Proper management of people with obesity during the COVID-19 pandemic. J Obes Metab Syndr 2020;29:84-98.

34. Hotamisligil GS, Shargill NS, Spiegelman BM. Adipose expression of tumor necrosis factor-alpha: direct role in obesitylinked insulin resistance. Science 1993;259:87-91.

35. Hivert MF, Sullivan LM, Fox CS, Nathan DM, D'Agostino RB Sr, Wilson PW, et al. Associations of adiponectin, resistin, and tumor necrosis factor-alpha with insulin resistance. J Clin 
Endocrinol Metab 2008;93:3165-72.

36. Berg AH, Scherer PE. Adipose tissue, inflammation, and cardiovascular disease. Circ Res 2005;96:939-49.

37. Illán-Gómez F, Gonzálvez-Ortega M, Orea-Soler I, Alcaraz-
Tafalla MS, Aragón-Alonso A, Pascual-Díaz M, et al. Obesity and inflammation: change in adiponectin, $\mathrm{C}$-reactive protein, tumour necrosis factor-alpha and interleukin- 6 after bariatric surgery. Obes Surg 2012;22:950-5. 